Revta brasil. Bot., São Paulo, V.24, n.1, p.51-62, mar. 2001

\title{
Fitossociologia de um remanescente de mata sobre tabuleiros no norte do estado do Rio de Janeiro (Mata do Carvão) ${ }^{1}$
}

\author{
GUILHERME C. DA SILVA² e MARCELO T. NASCIMENTO ${ }^{2,3}$
}

(recebido: 28 de julho de 1999; aceito: 4 de outubro de 2000)

\begin{abstract}
Phytosociological studies in a remnant lowland forest on tertiary "tabuleiros" in the north Rio de Janeiro State). The forests on Barreiras formations are located in fragmented areas extending from the Rio Grande do Norte State to north of Rio de Janeiro State. In the north of Rio de Janeiro State, the "Mata do Carvão" (1053 ha) is the largest remaining fragment. This study describes the structure and floristic composition of a remnant forest on tertiary tabuleiros and its main aim was to compare this forest with other "tabuleiro" and Atlantic forest remnants in the region. To carry out the inventory, four plots of $50 \times 50 \mathrm{~m}$ were allocated in a selected area without vestiges of logging activities or fire. All trees $\geq 10 \mathrm{~cm}$ DBH were sampled and numbered with aluminium tags. Dead trees were measured but not tagged. A total of 564 trees was sampled being distributed within 34 families. The families with higher number of species were Leguminosae (18), Myrtaceae (8), and Euphorbiaceae (6). The most abundant families were Rutaceae (189), Leguminosae (97) and Euphorbiaceae (47). The species with higher cover index values were Metrodorea brevifolia, Paratecoma peroba and Pseudopiptadenia contorta. Although the "Mata do Carvão" showed lower tree diversity $\left(\mathrm{H}^{\prime}=3.21\right)$ than those found for other semi-deciduous seasonal forests, it has a high species similarity between the tabuleiro forests of South Bahia and North of Espírito Santo. In the "Mata do Carvão", the occurrence of rare species at risk of extinction (e.g. Paratecoma peroba) was observed. Furthermore, these are new occurrences for the Rio de Janeiro State (Centrolobium sclerophyllum and Polygala pulcherrima).
\end{abstract}

RESUMO - (Fitossociologia de um remanescente de mata sobre tabuleiros no norte do Estado do Rio de Janeiro). As formações florestais sobre tabuleiros terciários ocorrem hoje na forma de pequenos fragmentos desde o Rio Grande do Norte até o Rio de Janeiro. No norte fluminense, a Mata do Carvão (1.053 ha) é o maior remanescente. Este trabalho descreve a estrutura e a composição florística desta mata, tendo por objetivo compará-la com outras matas da região. Foram estabelecidas quatro parcelas de $50 \mathrm{~m}$ x $50 \mathrm{~m}$ em uma área selecionada, sem vestígios de corte e de fogo. Todas as árvores com DAP $\geq 10 \mathrm{~cm}$ foram amostradas e plaqueadas. Árvores mortas foram medidas mas não plaqueadas. Um total de 564 árvores foram amostradas. Foram encontradas 34 famílias, sendo as de maior número de espécies Leguminosae (18), Myrtaceae (8) e Euphorbiaceae (6). As famílias mais abundantes foram Rutaceae (189), Leguminosae (97) e Euphorbiaceae (47). As espécies com maior índice de valor de cobertura (IVC) foram Metrodorea brevifolia, Paratecoma peroba e Pseudopiptadenia contorta. Embora a Mata do Carvão tenha uma diversidade (H'=3,21 nats) menor que outras matas estacionais semidecíduas (ex. mata de tabuleiro de Linhares), ela possui uma alta similaridade de espécies arbóreas com as matas de tabuleiro do sul da Bahia e do norte do Espírito Santo. Na Mata do Carvão foi observada a ocorrência de espécies raras típicas de mata de tabuleiro, como Paratecoma peroba, Centrolobium sclerophyllum e Polygala pulcherrima (novas ocorrências para a flora fluminense).

Key words - Atlantic forest, forest fragments, floristic, forest conservation, tabuleiro forest

\section{Introdução}

Ao longo da costa leste do Brasil, dentro do domínio Atlântico, principalmente entre os estados da Bahia e Rio de Janeiro, ocorre um tipo florestal denominado de Mata dos Tabuleiros (Ruschi 1950, Rizzini 1979). A denominação de tabuleiro é dada pelo fato da topografia apresentar-se plana em grandes extensões, não atingindo altitudes superiores a

1. Monografia apresentada por G.C. da Silva à UENF, para obtenção do título de Bacharel em Ciências Biológicas.

2. Universidade Estadual do Norte Fluminense (UENF), Laboratório de Ciências Ambientais (LCA), CBB, Av. Alberto Lamego 2000, 28015-620 Campos dos Goytacazes, RJ, Brasil.

3. Autor para correspondência: mtn@cbb.uenf.br
200 m (Rizzini 1979). Segundo RadamBrasil (1983), os sedimentos cenozóicos (terciários e quaternários) não são abundantes na área da mata atlântica, situando-se principalmente ao longo da orla marítima. Deles se destacam os depósitos arenosos e argiloarenosos de cores vivas do denominado Grupo (Formação) Barreiras, que ocorrem ao longo da costa, dos estados do Rio de Janeiro até o Pará, formando, em grandes extensões, planaltos costeiros de topos aplainados e terminando junto às praias por falésias abruptas, por vezes com mais de $20 \mathrm{~m}$ de altura, aos quais se atribui geralmente o nome de Tabuleiros.

As matas sobre os tabuleiros distinguem-se das outras formações de mata atlântica lato senso por ocuparem uma extensa área de planície ou tabuleiro costeiro, de origem terciária, com suas espécies distribuídas ao longo de um gradiente climático (sen- 
tido litoral-interior). Nestas matas, as espécies que ocorrem próximo do litoral, em geral, diferem daquelas que ocorrem no interior do continente, próximo às encostas das serras, enquanto nas demais formações que abrangem as matas serranas e suas encostas, as espécies se distribuem em um gradiente climático/topográfico (Rizzini 1979, Moreno et al. 1998). Outro aspecto que as tornam diferentes da mata atlântica de encosta (floresta ombrófila densa), é o fato de o interior da mata sobre os tabuleiros terciários, em geral, apresentar pouca vegetação rasteira e ocorrência de espécies epífitas (Ruschi 1950). A esclerofilia, típica das matas de tabuleiro, é outra característica diferencial (Rizzini 1979).

As matas sobre tabuleiros terciários sofreram uma intensa fragmentação, com o processo de desmatamento na costa brasileira durante a colonização e, mais recentemente (1960-1980) com a agricultura e com a industrialização (Rizzini 1979, Giulietti \& Forero 1990). Ainda hoje podem-se encontrar áreas de retirada ilegal de madeiras nestas formações. Com isso, restaram apenas alguns remanescentes florestais distribuídos ao longo da região costeira dos estados do nordeste até o estado do Rio de Janeiro. Entretanto, poucos são os estudos fitossociológicos realizados neste tipo florestal (Oliveira-Filho \& Carvalho 1993, Peixoto \& Gentry 1990, Peixoto et al. 1995, Rizzini et al. 1997), não sendo de nosso conhecimento nenhuma publicação sobre o assunto para as matas sobre tabuleiros da região norte Fluminense, principal área de ocorrência desta formação no estado do Rio de Janeiro.

Este trabalho descreve a estrutura e composição florística do componente arbóreo de um remanescente de mata sobre tabuleiros terciários do norte Fluminense, a Mata do Carvão, tendo por objetivo compará-la com outras matas de tabuleiros e também com outras matas de baixada da região.

\section{Material e métodos}

Área de estudo - Este estudo foi realizado em um remanescente de mata sobre tabuleiro terciário da região norte do estado do Rio de Janeiro (Mata do Carvão), localizado na Fazenda São Pedro ( $21^{\circ} 24^{\prime}$ S e $41^{\circ} 04^{\prime}$ W), município de São Francisco do Itabapoana, RJ, a $30 \mathrm{~km}$ em linha reta de Campos dos Goytacazes e a $10 \mathrm{~km}$ do litoral (figura 1). A precipitação média anual na região, no período de 1931 a 1960, foi de $1084 \mathrm{~mm}$ (RadamBrasil 1983), apresentando uma marcante sazonalidade, com um período seco de maio a setembro. Dados mais recentes (1984 a 1999) indicam

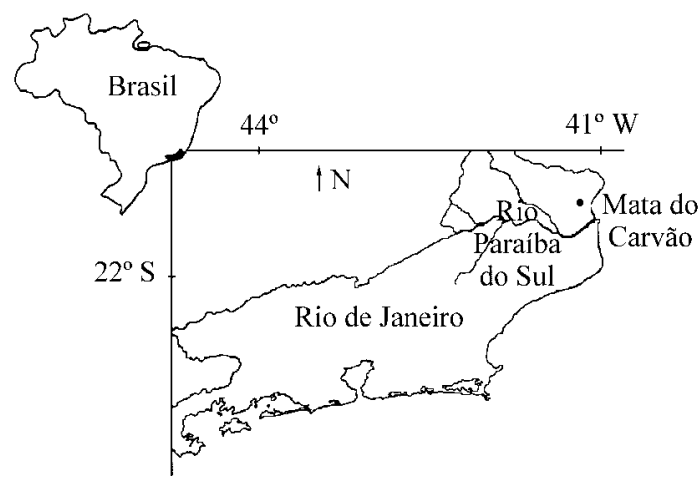

Figura 1. Mapa da localização da área de estudo, Mata do CarvãoRJ.

um período mais seco com uma precipitação média anual de 1023 $\mathrm{mm}$ (fonte: Estação Evapotranspirometrica UENF-PESAGRO, Campos dos Goytacazes). O solo da região é classificado como Podzólico Amarelo Álico de alta granulometria, baixa capacidade de retenção de água e pobre em nutrientes (RadamBrasil 1983).

A Mata do Carvão teve sua área reduzida aos atuais 1.053 ha devido aos desmatamentos ocorridos, principalmente durante as décadas de 60 e 70, para as atividades de plantio de cana-deaçúcar, pecuária, produção de carvão vegetal e comercialização de suas madeiras (por ex., Aspidosperma spp., Copaifera lucens, Paratecoma peroba e Tabebuia spp.). Segundo moradores da região, esses desmatamentos ocasionaram uma alteração na forma da mata, ficando esta distribuída em uma longa faixa de cerca de $5 \mathrm{~km}$ de comprimento e $2 \mathrm{~km}$ de largura, cortada por trilhas usadas na retirada de madeiras. Esta forma alongada, sem dúvida, acentua o efeito de borda nesta mata. Hoje, embora a taxa de desmatamento tenha diminuído, ainda ocorrem retiradas seletivas de madeira para a produção de lenha, cabos de ferramentas e/ou moirões em alguns pontos desta mata.

Através dos vestígios de corte e de fogo e com ajuda dos moradores locais, foram identificadas áreas com diferentes graus de perturbação, o que permitiu delimitar trechos que sofreram corte seletivo recentemente até trechos sem sinais de corte. O presente estudo foi realizado em um trecho de mata sem vestígios de corte ou de ocorrência de queimadas. Durante o estudo, embora tenha sido constatada a entrada de caçadores e lenhadores em áreas vizinhas, nenhuma árvore foi cortada nas parcelas estudadas.

Levantamento florístico e estrutural - Dentro da área escolhida de 25 ha, foi feito um sorteio de quatro pontos ao longo de uma trilha de $500 \mathrm{~m}$ para o estabelecimento de parcelas de $50 \mathrm{~m} \times 50 \mathrm{~m}$ $\left(2.500 \mathrm{~m}^{2}\right)$, sendo duas no sentido NE e duas no sentido SW da trilha, totalizando $1 \mathrm{ha}$, distando ca. $600 \mathrm{~m}$ da borda da mata e pelo menos $20 \mathrm{~m}$ da trilha. Em cada parcela, todas as árvores que possuíam diâmetro a altura do peito $(\mathrm{DAP}=$ diâmetro a $1,3 \mathrm{~m}$ do solo) maior ou igual a $10 \mathrm{~cm}$ foram marcadas com placas de alumínio numeradas. Indivíduos perfilhados só foram marcados e medidos quando um dos ramos possuía DAP $\geq 10 \mathrm{~cm}$. Árvores com raízes tabulares acima de $1,3 \mathrm{~m}$ tiveram seus diâmetros medidos $20 \mathrm{~cm}$ acima das raízes. Árvores mortas, tanto em pé quanto tombadas, foram medidas, mas não entraram nas análises 
dos dados florísticos e fitossociológicos por dificuldades na identificação botânica. Assim, só foram utilizadas como dados estruturais.

Durante a amostragem, foi coletado material fértil e/ou vegetativo para posterior identificação. Todo material coletado foi depositado na herbário do Laboratório de Ciências Ambientais (em implantação) da Universidade Estadual do Norte Fluminense (UENF) e as duplicatas enviadas ao herbário do Jardim Botânico do Rio de Janeiro (RB). A identificação do material foi feita parte na UENF e parte junto aos especialistas do Jardim Botânico do Rio de Janeiro.

Análise dos dados - Para a análise dos dados fitossociológicos, fo usado o programa FITOPAC (Shepherd 1995). Os parâmetros considerados foram: área basal total e individual (Abt e Abi), densidade relativa (DR), dominância relativa (DoR) e índice de valor de cobertura (IVC) (Brower \& Zar 1977). Para o cálculo da diversidade de famílias ou espécies, foi utilizado o índice de diversidade de Shannon (H') e o índice de equabilidade (J), calculados utilizando-se logaritmo natural. A similaridade de espécies entre parcelas foi calculada pelo índice de Morisita (Brower \& Zar 1977)

\section{Resultados}

Estrutura da mata - O número de indivíduos amostrados por parcela variou de 131 a 155 e a área basal de 3,2 a 4,3 $\mathrm{m}^{2}$ (tabela 1). A maior densidade de árvores ocorreu na classe de 10-15 cm de DAP, com poucas árvores atingindo DAP superiores a $40 \mathrm{~cm}$ (figura 2). Um baixo número de árvores perfilhadas $(n=9)$ foi amostrado, perfazendo $1,6 \%$ do total de indivíduos amostrados.

Embora a distribuição dos indivíduos em classes diamétricas incluindo todas as árvores amostradas na Mata do Carvão (figura 2) tenha apresentado um padrão de distribuição em forma de $\mathbf{J}$ invertido, sugerindo ausência de problemas de regeneração, uma análise mais detalhada, em nível específico, indicou que algumas espécies (ex.
Paratecoma peroba) possuíam padrões de distribuição distintos.

Um total de 70 árvores mortas (11\% do total amostrado) foi encontrado nas quatro parcelas estudadas (1 ha), com um DAP médio e desvio padrão de $19,5 \mathrm{~cm} 1,8 \mathrm{~cm}$. Entre estas, 61 estavam em pé, oito tombadas e uma quebrada (tabela 1).

Aspectos florísticos e fitossociológicos - Os 564 indivíduos amostrados (excluídos os mortos) foram distribuídos em 34 famílias (tabela 2), sendo as famílias Leguminosae e Myrtaceae as que apresentaram a maior riqueza de espécies. Destes, um total de 535 indivíduos foi identificado ao nível de gênero e/ou espécie (95\%), com 29 indivíduos (5\%), pertencentes a 12 morfoespécies, ainda indeterminados. Das 83 morfoespécies, foram identificadas 71 ao nível de gênero (85\%) e 56 (67\%) ao nível de espécie (tabela 3). Entre as espécies, 37 (44\%) ocorreram com apenas um indivíduo.

Entre as 83 espécies arbóreas amostradas nas parcelas (tabela 3 ), três delas podem ser consideradas novas ocorrências para a flora fluminense: Centrolobium sclerophyllum, Polygala pulcherrima e Trigoniodendron spiritusanctense.

Doze espécies foram comuns às quatro parcelas amostradas, representando $14 \%$ do total de espécies amostradas. Entre elas, destacam-se: Metrodorea brevifolia, Trichilia pseudostipulares e Pseudopiptadenia contorta (tabela 3). Destas, seis (Metrodorea brevifolia, Paratecoma peroba, Pseudopiptadenia contorta, Trichilia pseudostipulares, Talisia coriacea e Acacia polyphyla) somaram juntas ca. $50 \%$ do $\operatorname{IVC}(103,4)$ (tabela 4).

Diversidade, riqueza e similaridade - Os índices de diversidade de Shannon encontrados foram de

Tabela 1. Número de indivíduos, espécies e famílias (três indeterminadas), área basal, número de indivíduos mortos e DAP médio (desvio padrão) por parcela (0,25 ha) amostrada na Mata do Carvão, RJ.

\begin{tabular}{lcccccc}
\hline Parcelas (0,25 ha) & $\mathrm{n}^{\text {o }}$ de indivíduos & $\mathrm{n}^{\mathrm{o}}$ de espécies & $\mathrm{n}^{\mathrm{o}}$ de famílias & Área basal $\left(\mathrm{m}^{2}\right)$ & Mortas & DAP $(\mathrm{cm})$ \\
\hline P1 & 144 & 34 & 19 & 4,0 & 26 & $17,1 \pm 7,9$ \\
P2 & 131 & 45 & 22 & 3,2 & 18 & $16,5 \pm 6,2$ \\
P3 & 134 & 37 & 22 & 3,4 & 17 & $16,6 \pm 7,2$ \\
P4 & 155 & 50 & 22 & 4,3 & 9 & $17,2 \pm 7,9$ \\
Média & 141 & 41 & 21 & 3,7 & 17 & $16,8 \pm 7,3$ \\
\hline Total (1 ha) & 564 & 83 & 34 & 15,0 & 70 & \\
\hline
\end{tabular}



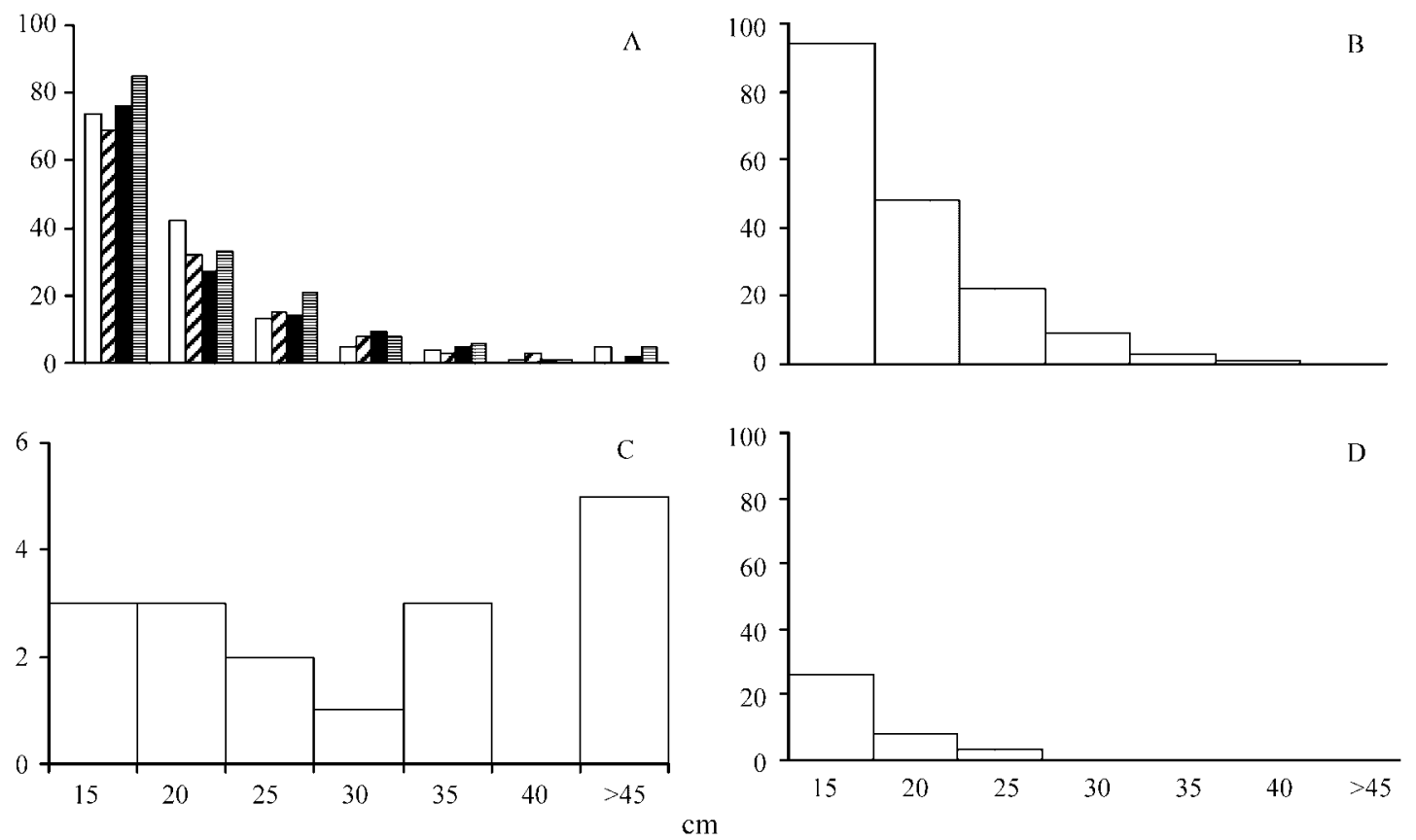

Figura 2. Distribuição diamétrica dos indivíduos com DAP $\geq 10 \mathrm{~cm}$ amostrados na Mata do Carvão (A) e para as três espécies de maior IVC, Metrodorea brevifolia (B, $\mathrm{n}=181)$, Paratecoma peroba $(\mathrm{C}, \mathrm{n}=17)$ e Pseudopiptadenia contorta $(\mathrm{D}, \mathrm{n}=36)$. ( $\square$ ) Parcela 1

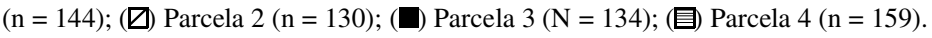

Tabela 2. Parâmetros fitossociológicos das 10 famílias de maior IVC amostradas nas quatro parcelas (1 ha) da Mata do Carvão. Nnúmero de indivíduos; Nsp- número de espécies; DR- densidade relativa; DoR- dominância relativa e IVC- índice de valor de cobertura.

\begin{tabular}{lrrrrr}
\hline Família & N & Nsp & DR $(\%)$ & DoR $(\%)$ & IVC \\
\hline Rutaceae & 189 & 4 & 33,51 & 29,53 & 63,04 \\
Leguminosae & 96 & 17 & 17,01 & 15,51 & 32,62 \\
$\quad$ Mimosoideae & 72 & 8 & 12,76 & 10,49 & 23,25 \\
Papilionoideae & 21 & 6 & 3,72 & 4,56 & 8,28 \\
$\quad$ Caesalpinioideae & 3 & 3 & 0,53 & 0,56 & 1,09 \\
Euphorbiaceae & 47 & 6 & 8,33 & 5,10 & 13,43 \\
Bignoniaceae & 23 & 3 & 4,08 & 9,24 & 13,32 \\
Meliaceae & 31 & 1 & 5,50 & 3,66 & 9,16 \\
Myrtaceae & 22 & 8 & 3,90 & 3,93 & 7,83 \\
Sapindaceae & 8 & 1 & 1,42 & 5,06 & 6,48 \\
Trigoniaceae & 14 & 1 & 2,48 & 2,38 & 4,86 \\
Cactaceae & 11 & 1 & 1,95 & 2,88 & 4,83 \\
Rubiaceae & 13 & 2 & 2,30 & 2,00 & 4,30 \\
Outras (n=24) & 110 & 39 & 19,52 & 23,59 & 40,13 \\
\hline Total & 564 & 100,00 & 100,00 & 200,00 \\
\hline
\end{tabular}


Tabela 3. Listagem de famílias e espécies, com nome vulgar e sua ocorrência nas parcelas amostradas na Mata do Carvão.

\begin{tabular}{|c|c|c|}
\hline Famílias / espécies & Nome vulgar & Parcelas \\
\hline \multicolumn{3}{|l|}{ ANACARDIACEAE } \\
\hline Astronium graveolens Jacq. & aderno & $\mathrm{P} 2$ \\
\hline Spondias sp. & & P3 \\
\hline \multicolumn{3}{|l|}{ ANNONACEAE } \\
\hline Oxandra nitida R.E. Fries & embiu-branco & $\mathrm{P} 1, \mathrm{P} 3, \mathrm{P} 4$ \\
\hline Oxandra sp. & embiu-preto & $\mathrm{P} 2, \mathrm{P} 4$ \\
\hline \multicolumn{3}{|l|}{ APOCYNACEAE } \\
\hline Aspidosperma illustre (Vell.) Kulhm. \& Pirajá & pequiá & $\mathrm{P} 1, \mathrm{P} 2$ \\
\hline Aspidosperma multiflorum A. DC. & caixão & $\mathrm{P} 2, \mathrm{P} 4$ \\
\hline \multicolumn{3}{|l|}{ BIGNONIACEAE } \\
\hline Paratecoma peroba (Record) Kuhlm. & peroba-do-campo & $\mathrm{P} 1, \mathrm{P} 2, \mathrm{P} 4$ \\
\hline Tabebuia serratifolia (Vahl) Nicholson & ipê-amarelo & $\mathrm{P} 1, \mathrm{P} 3, \mathrm{P} 4$ \\
\hline Tabebuia roseoalba (Rodley) Sandw. & ipê & P4 \\
\hline \multicolumn{3}{|l|}{ BOMBACACEAE } \\
\hline Eriotheca candolleana (K. Schum.) A. Robyns & mugumba & P4 \\
\hline Pseudobombax grandiflorum (Cav.) A. Robyns & imbiruçu & $\mathrm{P} 1, \mathrm{P} 3, \mathrm{P} 4$ \\
\hline \multicolumn{3}{|l|}{ BURSERACEAE } \\
\hline Protium sp. & gurubu & $\mathrm{P} 2, \mathrm{P} 4$ \\
\hline \multicolumn{3}{|l|}{ CACTACEAE } \\
\hline Opuntia brasiliensis (Willd.) Haw. & jurubeba & $\mathrm{P} 1, \mathrm{P} 2, \mathrm{P} 3, \mathrm{P} 4$ \\
\hline \multicolumn{3}{|l|}{ CARICACEAE } \\
\hline Jacaratia heptaphyla (Vell.) DC. & mamão-jacaratiá & P3 \\
\hline \multicolumn{3}{|l|}{ CELASTRACEAE } \\
\hline Maytenus brasiliensis Mart. & fruta-de-papagaio & $\mathrm{P} 1, \mathrm{P} 3, \mathrm{P} 4$ \\
\hline Maytenus sp. & fruta-de-papagaio & $\mathrm{P} 2$ \\
\hline \multicolumn{3}{|l|}{ CHRYSOBALANACEAE } \\
\hline Parinarisp. & bafo-de-boi & $\mathrm{P} 2$ \\
\hline \multicolumn{3}{|l|}{ COMBRETACEAE } \\
\hline Terminalia acuminata (Fr. All.) Eichl. & jundiaí & P4 \\
\hline \multicolumn{3}{|l|}{ ERYTHROXYLACEAE } \\
\hline Erythroxylum cuspidifolium Mart. & caga-fede & $\mathrm{P} 1, \mathrm{P} 2, \mathrm{P} 4$ \\
\hline \multicolumn{3}{|l|}{ EUPHORBIACEAE } \\
\hline Hyeronima oblonga (Tul.) Muell. Arg. & & P4 \\
\hline Joannesia princeps Vell. & induaçu & $\mathrm{P} 2, \mathrm{P} 3, \mathrm{P} 4$ \\
\hline Pachystroma longifolium (Nees) I.M. Johnston & gumané & $\mathrm{P} 1, \mathrm{P} 2, \mathrm{P} 3, \mathrm{P} 4$ \\
\hline Senefeldera multiflora Mart. & guarapoca & $\mathrm{P} 1, \mathrm{P} 2, \mathrm{P} 3, \mathrm{P} 4$ \\
\hline Euphorbiaceae 1 & & P3 \\
\hline \multicolumn{3}{|l|}{ FLACOURTIACEAE } \\
\hline Casearia sylvestris $\mathrm{Sw}$. & arco-de-pipa & $\mathrm{P} 2$ \\
\hline \multicolumn{3}{|l|}{ LAURACEAE } \\
\hline Ocotea sp. & pimenta-preta & $\mathrm{P} 2$ \\
\hline
\end{tabular}


(cont.)

\begin{tabular}{|c|c|c|}
\hline Famílias / espécies & Nome vulgar & Parcelas \\
\hline \multicolumn{3}{|l|}{ LECYTHIDACEAE } \\
\hline Cariniana legalis (Mart.) Kuntze & jequitibá & P3 \\
\hline Lecythis pisonis Camb. subsp. pisonis & sapucaia & $\mathrm{P} 1, \mathrm{P} 2, \mathrm{P} 3$ \\
\hline Lecythis lurida (Miers) Mori & inhaíba & $\mathrm{P} 2$ \\
\hline Couratari macrosperma A.C. Smith & ibirema & $\mathrm{P} 2$ \\
\hline Lecythidaceae 1 & & $\mathrm{P} 2$ \\
\hline \multicolumn{3}{|l|}{ LEGUMINOSAE } \\
\hline \multicolumn{3}{|l|}{ Mimosoideae } \\
\hline Acacia polyphylla (DC.) Benth. & monjolo-teta-de-porca & $\mathrm{P} 1, \mathrm{P} 2, \mathrm{P} 3, \mathrm{P} 4$ \\
\hline Albizia polycephala (Benth.) Killip & monjolo-alho & $\mathrm{P} 2, \mathrm{P} 3$ \\
\hline Parapiptadenia pterosperma (Benth.) Brenan & monjolo-branco & $\mathrm{P} 1, \mathrm{P} 2, \mathrm{P} 3, \mathrm{P} 4$ \\
\hline Pseudopiptadenia contorta (DC.) Lewis \& M. Lima & monjolo-sabão & $\mathrm{P} 1, \mathrm{P} 2, \mathrm{P} 3, \mathrm{P} 4$ \\
\hline Leguminosae 1 & & P1 \\
\hline Leguminosae 2 & & P4 \\
\hline Leguminosae 3 & & P4 \\
\hline \multicolumn{3}{|l|}{ Papilionoideae } \\
\hline Acosmium lentiscifolium Spreng. & moço-branco & $\mathrm{P} 1, \mathrm{P} 4$ \\
\hline Centrolobium sclerophyllum H. C. Lima & Araribá & $\mathrm{P} 2$ \\
\hline Machaerium incorruptibile (Vell.) Fr. All. ex Benth. & jacarandá-cipó & $\mathrm{P} 1, \mathrm{P} 2, \mathrm{P} 3$ \\
\hline Machaerium sp. & & P3 \\
\hline Myrocarpus frondosus Fr. All. & óleo-vermelho & $\mathrm{P} 1$ \\
\hline Platymiscium floribundum Vog. & & P1 \\
\hline Platipodium elegans Vog. & sucupira & $\mathrm{P} 2$ \\
\hline \multicolumn{3}{|l|}{ Caesalpinioideae } \\
\hline Caesalpinea ferrea Mart. & pau-ferro & P4 \\
\hline Copaifera lucens Dwyers & copaíba & $\mathrm{P} 2$ \\
\hline Peltogyne discolor Vog. & roxinho & P2 \\
\hline \multicolumn{3}{|l|}{ MALPIGHIACEAE } \\
\hline Byrsonima sp. & & P4 \\
\hline \multicolumn{3}{|l|}{ MELIACEAE } \\
\hline Trichilia pseudostipulares (A. Juss.) DC. & óleo-de-marceneiro & $\mathrm{P} 1, \mathrm{P} 2, \mathrm{P} 3, \mathrm{P} 4$ \\
\hline \multicolumn{3}{|l|}{ MORACEAE } \\
\hline Brosimum guianense (Aubl.) Huber & leiteira & P3 \\
\hline \multicolumn{3}{|l|}{ MYRTACEAE } \\
\hline Eugenia sp. 1 & araça & $\mathrm{P} 1, \mathrm{P} 2, \mathrm{P} 3$ \\
\hline Eugenia sp. 2 & & $\mathrm{P} 2$ \\
\hline Myrcia sp. & & P4 \\
\hline Myrtaceae 1 & & $\mathrm{P} 3, \mathrm{P} 4$ \\
\hline Myrtaceae 2 & & $\mathrm{P} 2$ \\
\hline Myrtaceae 3 & & P4 \\
\hline Plinia cauliflora (Mart.) Kausel & jaboticabeira & P3 \\
\hline
\end{tabular}


(cont.)

\begin{tabular}{|c|c|c|}
\hline Famílias / espécies & Nome vulgar & Parcelas \\
\hline Syzygium jambos (L.) Alston & jambo & $\mathrm{P} 4$ \\
\hline \multicolumn{3}{|l|}{ NYCTAGINACEAE } \\
\hline Andradea floribunda Fr. All. & siriba & $\mathrm{P} 1, \mathrm{P} 4$ \\
\hline Guapira opposita (Vell.) Reitz & maria-mole & $\mathrm{P} 3, \mathrm{P} 4$ \\
\hline \multicolumn{3}{|l|}{ OLACACEAE } \\
\hline Heisteria sp. & fruto-de-papagaio & $\mathrm{P} 1$ \\
\hline \multicolumn{3}{|l|}{ POLYGALACEAE } \\
\hline Polygala pulcherrima Kuhlm. & & $\mathrm{P} 3$ \\
\hline \multicolumn{3}{|l|}{ POLYGONACEAE } \\
\hline Coccoloba alnifolia Casar. & folha-de-bolo & $\mathrm{P} 2, \mathrm{P} 4$ \\
\hline Coccoloba sp. & folha-de-bolo & $\mathrm{P} 1, \mathrm{P} 4$ \\
\hline \multicolumn{3}{|l|}{ RUBIACEAE } \\
\hline Alseis pickelli Pilger et Schmale & goiabeira & $\mathrm{P} 1, \mathrm{P} 2, \mathrm{P} 3, \mathrm{P} 4$ \\
\hline Amaioua intermedia var. brasiliana (A. Rich.) Steyerm. & & $\mathrm{P} 4$ \\
\hline Coutarea hexandra (Jacq.) K. Schum. & & P4 \\
\hline \multicolumn{3}{|l|}{ RUTACEAE } \\
\hline Metrodorea brevifolia Engl. & goroeitá & $\mathrm{P} 1, \mathrm{P} 2, \mathrm{P} 3, \mathrm{P} 4$ \\
\hline Neoraputia alba (Nees \& Mart.) Emmerich & guaratáia & $\mathrm{P} 1, \mathrm{P} 2, \mathrm{P} 4$ \\
\hline Zanthoxylum rhoifolium Lam. & laranjinha & $\mathrm{P} 1$ \\
\hline Rutaceae 1 & & $\mathrm{P} 2$ \\
\hline \multicolumn{3}{|l|}{ SAPINDACEAE } \\
\hline Talisia coriacea Radlk. & pitomba & P1, P2, P3, P4 \\
\hline \multicolumn{3}{|l|}{ SAPOTACEAE } \\
\hline Chrysophyllum lucentifolium Cronq. & jacoá & P1, P2, P3, P4 \\
\hline \multicolumn{3}{|l|}{ SOLANACEAE } \\
\hline Metternichia princeps Mikan & cafezinho & $\mathrm{P} 1, \mathrm{P} 2, \mathrm{P} 3$ \\
\hline \multicolumn{3}{|l|}{ STERCULIACEAE } \\
\hline Guazuma ulmifolia Lam. & algodão & $\mathrm{P} 3$ \\
\hline Pterigota brasiliensis Fr. All. & farinha-seca & P3 \\
\hline \multicolumn{3}{|l|}{ TILIACEAE } \\
\hline Luehea divaricata Mart. & saco-de-gambá & $\mathrm{P} 2, \mathrm{P} 3$ \\
\hline \multicolumn{3}{|l|}{ TRIGONIACEAE } \\
\hline Trigoniodendron spiritusanctense Guim. \& Miguel & milho-cozido & $\mathrm{P} 1, \mathrm{P} 2, \mathrm{P} 3, \mathrm{P} 4$ \\
\hline \multicolumn{3}{|l|}{ ULMACEAE } \\
\hline Celtis sp. & limão & $\mathrm{P} 1, \mathrm{P} 2, \mathrm{P} 4$ \\
\hline \multicolumn{3}{|l|}{ Indeterminadas } \\
\hline Indeterminada 1 & & $\mathrm{P} 4$ \\
\hline Indeterminada 2 & & $\mathrm{P} 4$ \\
\hline Indeterminada 3 & & $\mathrm{P} 1, \mathrm{P} 2, \mathrm{P} 3$ \\
\hline
\end{tabular}


Tabela 4. Parâmetros fitossociológicos das 35 espécies de maior IVC amostradas nas quatro parcelas (1 ha) da Mata do Carvão. $\mathrm{N}$ - número de indivíduos; AB - área basal; DR - densidade relativa; DoR - dominância relativa e IVC - índice de valor de cobertura.

\begin{tabular}{|c|c|c|c|c|c|}
\hline Espécie & $\mathrm{N}$ & $\mathrm{AB}\left(\mathrm{m}^{2}\right)$ & $\operatorname{DR}(\%)$ & $\operatorname{DoR}(\%)$ & IVC \\
\hline Metrodorea brevifolia & 181 & 4,13 & 32,09 & 27,61 & 59,70 \\
\hline Paratecoma peroba & 17 & 1,26 & 3,01 & 8,47 & 11,48 \\
\hline Pseudopiptadenia contorta & 36 & 0,57 & 6,38 & 3,85 & 10,23 \\
\hline Trichilia pseudostipulares & 31 & 0,54 & 5,50 & 3,66 & 9,16 \\
\hline Talisia coriacea & 8 & 0,75 & 1,42 & 5,06 & 6,48 \\
\hline Acacia polyphyla & 22 & 0,36 & 3,90 & 2,47 & 6,37 \\
\hline Eugenia sp. 1 & 14 & 0,45 & 2,48 & 3,06 & 5,54 \\
\hline Parapiptadenia pterosperma & 9 & 0,49 & 1,60 & 3,28 & 4,88 \\
\hline Trigoniodrendron spiritusanctense & 14 & 0,35 & 2,48 & 2,38 & 4,86 \\
\hline Opuntia brasiliensis & 11 & 0,43 & 1,95 & 2,88 & 4,83 \\
\hline Hyeronima oblonga & 14 & 0,25 & 2,48 & 1,73 & 4,21 \\
\hline Alseis pickelli & 12 & 0,28 & 2,13 & 1,91 & 4,04 \\
\hline Indeterminada 3 & 9 & 0,27 & 1,60 & 1,83 & 3,43 \\
\hline Metternichia principes & 13 & 0,15 & 2,30 & 1,06 & 3,36 \\
\hline Pseudobombax grandiflorum & 5 & 0,34 & 0,89 & 2,28 & 3,17 \\
\hline Pachystroma longifolium & 12 & 0,12 & 2,13 & 0,85 & 2,98 \\
\hline Machaerium incorruptibile & 7 & 0,23 & 1,24 & 1,57 & 2,81 \\
\hline Maytenus brasiliensis & 8 & 0,19 & 1,42 & 1,30 & 2,72 \\
\hline Neoraputia alba & 6 & 0,21 & 1,06 & 1,42 & 2,48 \\
\hline Indeterminada 1 & 8 & 0,15 & 1,42 & 1,06 & 2,48 \\
\hline Senefeldera multiflora & 9 & 0,11 & 1,60 & 0,75 & 2,35 \\
\hline Chrysophilum lenticifolium & 8 & 0,11 & 1,42 & 0,73 & 2,15 \\
\hline Andradea floribunda & 2 & 0,26 & 0,35 & 1,78 & 2,13 \\
\hline Acosmium lentiscifolium & 5 & 0,13 & 0,89 & 0,93 & 1,82 \\
\hline Oxandra nitida & 6 & 0,09 & 1,06 & 0,62 & 1,68 \\
\hline Pterigota brasiliensis & 2 & 0,19 & 0,35 & 1,33 & 1,68 \\
\hline Celtis sp. & 4 & 0,13 & 0,71 & 0,91 & 1,62 \\
\hline Tabebuia serratifolia & 5 & 0,10 & 0,89 & 0,67 & 1,56 \\
\hline Myrocarpus frondosus & 5 & 0,07 & 0,89 & 0,47 & 1,36 \\
\hline Coccoloba alnifolia & 4 & 0,09 & 0,71 & 0,62 & 1,33 \\
\hline Erythroxylum cuspidiforme & 5 & 0,05 & 0,89 & 0,36 & 1,25 \\
\hline Protium sp. & 2 & 0,12 & 0,35 & 0,83 & 1,18 \\
\hline Joannesia principes & 3 & 0,09 & 0,53 & 0,63 & 1,16 \\
\hline Oxandra sp. & 4 & 0,06 & 0,71 & 0,45 & 1,16 \\
\hline Coccoloba sp. & 3 & 0,06 & 0,53 & 0,50 & 1,09 \\
\hline Outras (48 spp.) & 60 & 1,82 & 10,64 & 10,69 & 21,27 \\
\hline Total & 564 & 15 & 100,00 & 100,00 & 200 \\
\hline
\end{tabular}


2,6 nats para famílias e de 3,21 nats para espécies, com um valor da equabilidade de 0,77. Entre as parcelas, os valores de diversidade de espécies variaram de 2,66 nats a 3,15 nats, com os valores dos índices de similaridade de espécies arbóreas variando entre 0,53 e 0,62 .

\section{Discussão}

Estrutura da mata - Os valores de DAP médio das árvores nas parcelas e de área basal total $(16,8 \mathrm{~cm} \pm$ 0,34 d.p. e $15 \mathrm{~m}^{2} \cdot \mathrm{ha}^{-1}$ ) foram baixos quando comparados com outras matas estacionais semideciduais, tais como a mata de tabuleiro de Linhares e com a floresta estacional decidual de Peltogyne em Maracá, RR $\left(24 \mathrm{~cm}\right.$ e $33 \mathrm{~m}^{2} \cdot \mathrm{ha}^{-1}$, Nascimento et al. 1997), e menor ainda quando comparada com a mata atlântica de encosta no Imbé, RJ ( $26 \mathrm{~cm}$ e $40 \mathrm{~m}^{2}$.ha ${ }^{-1}$, Moreno et al. 1998). Este fato parece estar relacionado não só ao tipo de mata, mas muito provavelmente ao grau de perturbação antrópica da área. Um baixo valor de área basal $\left(18 \mathrm{~m}^{2} \cdot \mathrm{ha}^{-1}\right)$ também foi encontrado para uma mata atlântica de baixada na região Norte Fluminense (M.T. Nascimento, dados não publicados). Esta mata, assim como a Mata do Carvão, teve sua área bem reduzida no passado em função de desmatamentos para plantio de monocultura da cana-de-açúcar e de pastagens e outros impactos antrópicos, como a retirada de lenha e o corte seletivo, seguindo o padrão geral de degradação observado para as matas do domínio atlântico (Giulietti \& Forero 1990). Assim, o alto número de árvores com DAP entre 10 e $20 \mathrm{~cm}$ e o baixo valor de área basal total por hectare, muito provavelmente, são reflexo destas perturbações.

O fato das árvores da Mata do Carvão, na amostragem geral, apresentarem um padrão de distribuição diamétrica em forma de $\mathrm{J}$ invertido não necessariamente indica ausência de problemas de regeneração, devendo ser considerado com cautela. Outros estudos (Martins 1991, Santos et al. 1998) têm demonstrado a necessidade de uma análise mais detalhada, ao nível específico, e com um grupo maior de espécies.

O número de árvores mortas em pé (61 em 625 árvores amostradas) pode ser considerado alto se comparado com os ca. $3 \%$ citados para outras matas no estado do Rio de Janeiro (8 em 592 indivíduos -
Kurtz 1994, 7 em 460 indivíduos - M.R. Moreno, dados não publicados). Este resultado, provavelmente, está relacionado ao grau de perturbação (fragmentação e efeito de borda) que a Mata do Carvão tem sofrido.

Aspectos florísticos e fitossociológicos - As famílias Leguminosae e Myrtaceae, que apresentaram a maior riqueza de espécies arbóreas na Mata do Carvão, são as que, em geral, ocorrem com o maior número de espécies ao longo da costa atlântica brasileira (Mori et al. 1983 e Peixoto \& Gentry 1990). Gentry (1982) considera que a família Leguminosae está entre as mais ricas em espécies em matas neotropicais de baixada.

A ocorrência de Metrodorea brevifolia, tão comum na Mata do Carvão e na mata da Emerência, Cabo Frio, RJ (Farág 1999), porém rara ou ausente em outras matas estacionais semideciduais como as de Linhares (Peixoto \& Gentry 1990, Rizzini et al. 1997) e de Caratinga (Oliveira-Filho \& Fontes 2000), sugere ser esta espécie característica das matas estacionais do litoral norte fluminense.

Martins (1991), estudando uma mata semidecídua e Salis et al. (1994), estudando uma mata ciliar, ambas no estado de São Paulo, encontraram como espécie mais abundante Metrodorea nigra, que semelhante a M. brevifolia, ocorre com maior frequiência no estrato inferior destas matas. Segundo Martins (1991), a destruição seletiva de indivíduos de fustes grossos deve ter sido um dos fatores que contribuíram para o aumento na densidade de espécies como M. nigra, fato que muito provavelmente também deve ter acontecido na Mata do Carvão. A ocorrência de espécies típicas de estrato intermediário e/ou inferior, tais como Metrodorea brevifolia, Trichilia pseudostipularis e Pseudopiptadenia contorta, como as de maior abundância e dominando o dossel e a ocorrência em densidades baixas ou mesmo ausência de espécies típicas do dossel (ex: Aspidosperma polyneuron), que segundo moradores da região eram comuns nesta mata e foram intensamente exploradas entre as décadas de 60 e 80, indicam que esta mata é secundária e sofreu uma perda de diversidade arbórea no dossel.

Em relação à composição florística, foi observado que das 56 espécies identificadas nas parcelas estudadas, 34 (62\%) constam na lista de espécies que 
ocorrem em Linhares (Jesus \& Garcia 1992) (tabela 5). Este elevado número de espécies comuns deve, muito provavelmente, ser ainda maior, estando subestimado visto que na Mata do Carvão não foi ainda realizado um amplo levantamento florístico como já efetuado para as matas de Linhares. Espécies como Centrolobium sclerophyllum, Polygala pulcherrima e Trigoniodendron spiritusanctense, novas ocorrências para a flora fluminense e antes citadas como endêmicas de Linhares, podem ser consideradas características deste tipo florestal.

O baixo índice pluviométrico anual da Mata do Carvão, aliado à forte sazonalidade, com um inverno seco (maio até setembro), pode ser considerado um dos fatores responsáveis pela maior ocorrência nesta mata de espécies decíduas tais como Paratecoma peroba, Myrocarpus frondosus, Tabebuia spp., Machaerium incorruptibile, entre outras. Aspectos do clima e a alta densidade destas espécies permitem classificar a Mata do Carvão como sendo uma formação estacional semidecidual.

Um número baixo de espécies comuns entre a Mata do Carvão e as áreas de floresta ombrófila

Tabela 5. Número e percentagem de espécies da Mata do Carvão que ocorrem em outras matas da região sudeste. Baseado nas 55 espécies identificadas para a Mata do Carvão.

\begin{tabular}{|c|c|c|c|}
\hline Matas & $\mathrm{N}^{\circ}$ de spp. & $\%$ spp. & Fonte \\
\hline \multicolumn{4}{|l|}{ Matas semidecíduas } \\
\hline Linhares (ES) & 34 & 62 & $\begin{array}{l}\text { Jesus \& Garcia } \\
\text { (1992) }\end{array}$ \\
\hline Cabo Frio (RJ) & 24 & 44 & Araujo et al.(1998) \\
\hline Mergulhão (RJ) & 16 & 29 & $\begin{array}{l}\text { M. T. Nascimento } \\
\text { (dados não } \\
\text { publicados) }\end{array}$ \\
\hline Caratinga (MG) & 25 & 45 & $\begin{array}{l}\text { Oliveira-Filho \& } \\
\text { Fontes }(2000)\end{array}$ \\
\hline Rio Doce (MG) & 19 & 34 & $\begin{array}{l}\text { Oliveira-Filho \& } \\
\text { Fontes }(2000)\end{array}$ \\
\hline Viçosa (MG) & 15 & 27 & $\begin{array}{l}\text { Oliveira-Filho \& } \\
\text { Fontes (2000) }\end{array}$ \\
\hline \multicolumn{4}{|l|}{ Matas Ombrófilas } \\
\hline Imbé (RJ) & 6 & 11 & $\begin{array}{l}\text { Moreno et al. } \\
\text { (1998) }\end{array}$ \\
\hline $\begin{array}{l}\text { Juiz de Fora } \\
\text { (MG) }\end{array}$ & 7 & 13 & $\begin{array}{l}\text { Oliveira-Filho \& } \\
\text { Fontes (2000) }\end{array}$ \\
\hline $\begin{array}{l}\text { Ponte Nova } \\
(\mathrm{MG})\end{array}$ & 3 & 5 & $\begin{array}{l}\text { Oliveira-Filho \& } \\
\text { Fontes }(2000)\end{array}$ \\
\hline
\end{tabular}

densa do estado do Rio de Janeiro foi observado. Como exemplos, têm-se Chrysophyllum lucentifolium, Senefeldera multiflora, Cariniana legalis, Guapira opposita e Oxandra nitida para a mata de Cachoeiras de Macacu-RJ (Kurtz 1994), Eriotheca candolleana, Maytenus brasiliensis, Erythroxylum cuspidifolium, Myrocarpus frondosus e Guapira opposita para a Reserva Ecológica de Macaé de Cima-RJ (Guedes-Bruni et al. 1997) e Pseudopiptadenia contorta e Guapira opposita, para a mata da Região do Imbé (Moreno et al. 1998). O número de espécies comuns foi menor que o encontrado para as matas estacionais semideciduais de Cabo Frio, RJ, Linhares, ES e Caratinga, MG (tabela 5), sugerindo que a proximidade geográfica não é o fator preponderante na composição florística da Mata do Carvão. Entre as espécies comuns à Mata do Carvão e às matas estacionais semidecíduas acima citadas, destacam-se Acosmium lentiscifolium, Astronium graveolens, Caesalpinea ferrea, Cariniana legalis, Eriotheca candoleana, Joannesia princeps, Myrocarpus frondosus, Pseudopiptadenia contorta e Senefeldera multiflora.

Oliveira-Filho \& Fontes (2000), após uma ampla análise florística baseada em dados de diversas localidades de mata atlântica da região sudeste, concluem que o norte fluminense representa uma interrupção na distribuição da floresta ombrófila densa (floresta pluvial), com a penetração até a costa da floresta semidecídua. Estes autores consideraram o clima, e em especial a temperatura, como o fator mais fortemente relacionado com as variações florísticas observadas.

Diversidade, riqueza e similaridade - Peixoto \& Gentry (1990) encontraram 99 indivíduos (DAP > 10 $\mathrm{cm}$ ) distribuídos em 65 espécies em uma amostra de 0,1 ha na mata de tabuleiro da Reserva Florestal de Linhares, ES. A área amostral de Linhares foi 10 vezes menor que a adotada na Mata do Carvão, mas apresentou uma maior riqueza de espécies. $\mathrm{O}$ fato da Mata do Carvão ter apresentado, proporcionalmente, menos espécies que a mata de Linhares, máximo de 50 em 0,25 ha contra os 65 em 0,1 ha encontrados para Linhares, pode estar relacionado a fatores como os efeitos de borda, devido à perda de área e a mudança na forma, ocorridos na Mata do Carvão. Embora a mata de Linhares possua locais com diferentes intensidades de efeito de borda, devido ao 
desenho da reserva e ao número de estradas internas, sua área é de ca. 21.000 ha, em sua maioria contínuos, enquanto que a Mata do Carvão possui hoje apenas $1.053 \mathrm{ha}$, distribuídos em uma faixa de $5 \mathrm{~km}$ x $2 \mathrm{~km}$ e cortada por trilhas utilizadas para o escoamento de madeiras. Segundo Turner \& Corlett (1996), os fragmentos florestais perdem espécies por fatores como a formação de um microclima propício para que espécies oportunistas possam competir por recursos com espécies nativas (efeito de borda) e a erosão gênica, que ocorre em populações geneticamente limitadas, reduzindo a variabilidade gênica nestas populações.

O índice de diversidade de Shannon para a Mata do Carvão ( $\mathrm{H}^{\prime}=3,21$ nats) foi próximo ou ligeiramente inferior aos encontrados por Cavassan et al. (1984) em Bauru (H'= 3,50 nats), Silva \& LeitãoFilho (1982) em Ubatuba (H' $=3,49$ nats) e M.T. Nascimento (dados não publicados) em Campos $\left(H^{\prime}=3,67\right.$ nats) para florestas estacionais da região sudeste. Entretanto, essas comparações devem ser consideradas com cautela em função de diferenças na metodologia utilizada, como por exemplo em relação ao número de árvores amostradas, ao critério de inclusão (DAP mínimo), entre outros, que são fatores que influenciam na avaliação da riqueza de espécies (Martins 1991). Greig-Smith (1983) apontou os efeitos do tamanho da parcela nas estimativas de diversidade e mais recentemente Laurance $e t$ al. (1998) alertaram para a influência da forma das parcelas em estimativas de diversidade e composição da comunidade.

A Mata do Carvão, o maior remanescente de mata de tabuleiro no estado do Rio de Janeiro, apresentou uma forte similaridade florística com as matas de tabuleiro do Norte do Espírito Santo, sugerindo uma maior ligação florística com essas matas do que com outras mais próximas geograficamente como as matas estacionais deciduais de Cabo Frio e Viçosa. Medidas concretas visando sua preservação devem ser implementadas, tais como sua transformação em uma Unidade de Conservação e o enriquecimento florestal a partir de plantios de mudas de espécies outrora abundantes (ex. Paratecoma peroba e Aspidosperma polyneuron) (Rizzini 1979, RadamBrasil 1983) e hoje raras em decorrência dos sucessivos cortes seletivos de madeira.
Agradecimentos - Aos pesquisadores Bruno Kurtz, Cyl Farney e Haroldo Lima (Instituto de Pesquisa do Jardim Botânico do Rio de Janeiro), João Marcelo A. Braga (UENF), Mário Gomes, Dorothy S. Araújo (UFRJ) e Ronaldo Marquete (IBGE), pelo auxílio na identificação do material coletado; à Flávio A. M. Santos pelas críticas ao manuscrito; aos proprietários da Fazenda São Pedro, Ricardo e Vera Mansur, por permitirem o livre acesso à área de estudo; à Fundação de Amparo a Pesquisa do Estado do Rio de Janeiro (FAPERJ) pelo apoio financeiro, à Fundação Estadual do Norte Fluminense (FENORTE) pelo apoio logístico e ao Conselho Nacional de Pesquisas e Tecnologia (CNPq) pelas bolsas de I.C. (PIBIC) à G.C. Silva e de Produtividade Científica à M.T. Nascimento.

\section{Referências bibliográficas}

ARAUJO, D.S., LIMA, H.C., FARÁG, P.R.C., LOBAO, A.Q., SA, C.F.C. \& KURTZ, B.C. 1998. O centro de diversidade vegetal de Cabo Frio: Levantamento preliminar da flora. In Anais do IV Simpósio de Ecossistemas Brasileiros, ACIESP, Águas de Lindóia, SP, v.II, p.147-157.

BROWER, J.E. \& ZAR, J.H. 1977. Field and laboratory methods for general ecology. W.C. Brown Company Publishers, Iowa.

CAVASSAN, O., CESAR, O. \& MARTINS, F.R. 1984. Fitossociologia da vegetação arbórea da Reserva Estadual de Bauru, Estado de São Paulo. Revista Brasileira de Botânica 7:91-106.

FARÁG, P.R.C. 1999. Estrutura do estrato arbóreo de mata litorânea semicaducifólia sobre solo arenoso no município de Búzios, RJ. Dissertação de mestrado, Museu Nacional, Universidade Federal do Rio de Janeiro, Rio de Janeiro.

GENTRY, A. 1982. Neotropical floristic diversity: phytogeographical connections between Central and South America, pleistocene climatic fluctuations, or an accident of the andean orogeny? Annals of Missouri Botanical Garden 69:557-593.

GIULIETTI, A.M. \& FORERO, E. 1990. "Workshop" diversidade taxonômica e padrões de distribuição das angiospermas brasileiras. Introdução. Acta Botanica Brasilica 4:3-9.

GUEDES- BRUNI, R.R., PESSOA, S.V.A. \& KURTZ, B.C. 1997. Florística e estrutura do componente arbustivoarbóreo de um trecho preservado de floresta montana na Reserva Ecológica de Macaé de Cima. In Serra da Macaé de Cima: diversidade florística e conservação em Mata atlântica (H.C. Lima \& R.R. Guedes-Bruni, eds.), Jardim Botânico, Rio de Janeiro, p.127-144.

GREIG-SMITH, P. 1983. Quantitative plant ecology. 3 ed. University of California Press, Berkeley.

JESUS, R.M. \& GARCIA, A. 1992. O Herbário da Reserva Florestal de Linhares. Anais do $2^{\circ}$ Congresso Nacional sobre Essências Nativas. Revista do Instituto Florestal 4:350-362. 
KURTZ, B.C. 1994. Composição florística e estrutura do componente arbóreo de um trecho de Mata Atlântica na Estação Ecológica Estadual do Paraíso, Cachoeiras de Macacu, RJ. Dissertação de mestrado, Universidade Federal do Rio de Janeiro, Rio de Janeiro.

LAURANCE, W.F., FERREIRA, L.V., RANKIN-DE MERONA \& HUTCHINGS, R. W. 1998. Influence of plot shape on estimates of tree diversity and community composition in central Amazonia. Biotropica 30:662-665.

MARTINS, F.R. 1991. Estrutura de uma floresta mesófila. Editora da UNICAMP, Campinas.

MORENO, M.R., NASCIMENTO, M.T. \& KURTZ, B. 1998. Estrutura e composição florística do estrato arbóreo em duas zonas altitudinais diferentes em Mata Atlântica de encosta na região do Imbé, RJ: primeira aproximação. In Anais do IV Simpósio de Ecossistemas Brasileiros. (S. Watanabe, coord.), ACIESP, v.II, p.64-70.

MORI, S.A., BOOM, B.M., CARVALINO, A.M. \& SANTO, T.S. 1983. Ecological importance of Myrtaceae in a eastern Brazilian wet forest. Biotropica 15:68-70.

NASCIMENTO, M.T., PROCTOR, J. \& VILLELA, D.M. 1997. Forest structure, floristic composition and soils of an Amazonian monodominant forest on Maraca Island, Roraima, Brazil. Edinburgh Journal of Botany 54:1-38.

OLIVEIRA-FILHO, A.T. \& CARVALHO, C. 1993. Florística e fisionomia da vegetação no extremo norte do litoral da Paraíba. Revista Brasileira de Botânica 16:115-130.

OLIVEIRA-FILHO, A.T. \& FONTES, M.A.L. 2000. Patterns of floristic differentiation among Atlantic forests in south-eastern Brazil, and the influence of climate. Biotropica 31 (no prelo).

PEIXOTO, A.L. \& GENTRY, A. 1990. Diversidade e composição florística da mata de tabuleiro na Reserva Florestal de Linhares (Espírito Santo, Brasil). Revista Brasileira de Botânica 13:19-25.
PEIXOTO, A.L., ROSA, M.M.T. \& JOELS, L.C.M. 1995. Diagramas de perfil e de cobertura de um trecho da Floresta de Tabuleiro na Reserva Florestal de Linhares (Espírito Santo, Brasil). Acta Botanica Brasilica 9:177-194.

RADAMBRASIL. 1983. Levantamento de recursos naturais, v.32, folha S/ F. 23/ 24. Rio de Janeiro/ Vitória. Ministério das Minas e Energia, Rio de Janeiro.

RIZZINI, C. T. 1979. Tratado de fitogeografia do Brasil. v.2. Aspectos ecológicos. Hucitec / Edusp, São Paulo.

RIZZINI, C., ADUAN, R.E., JESUS, R. \& GARAY, I. 1997. Floresta pluvial de tabuleiros, Linhares, ES, Brasil: sistemas primários e secundários. Leandra 12:54-76.

RUSCHI, A. 1950. Fitogeografia do Estado do Espírito Santo. v.l. Considerações gerais sobre a distribuição da flora do Estado do Espírito Santo. Boletim do Museu de Biologia Mello Leitão, Série Botânica, 1:1-353.

SALIS, S.M., TAMASHIRO, J.Y.\& JOLY, C.A.1994. Florística e fitossociologia do estrato arbóreo de um remanescente de mata ciliar do rio Jacaré-Pepira, Brotas, SP. Revista Brasileira de Botânica 17: 93-103.

SANTOS, F.A.M., PEDRONI, F., ALVES, L.F. \& SANCHEZ, M. 1998. Structure and dynamics of tree species of the Atlantic Forest. Anais da Academia Brasileira de Ciências 70:874-880.

SHEPHERD, G.J. 1995. FITOPAC. Manual do Usuário. UNICAMP, Campinas, SP.

SILVA, A.F. \& LEITÃO-FILHO, H.F. 1982. Composição florística e estrutura fitossociológica de um trecho de Mata Attântica de encosta no município de Ubatuba, São Paulo. Revista Brasileira de Botânica 5:43-52.

TURNER, I.M. \& CORLETT, R.T. 1996. The conservation value of small isolated fragments of lowland tropical rain forest. Trends in Ecology and Evolution 11:330-333. 